\title{
Planificación de la Operación de Corto Plazo de Sistemas de Energía Termoeléctrica con Restricciones Ambientales
}

João P. Catalão (1), Sílvio J. Mariano (1), Victor M. Mendes (2) y Luís A. Ferreira (3)

(1) Universidad Beira Interior, Dpto. de Ingeniaría Electromecánica,

Calle Fonte do Lameiro, 6201-001 Covilha-Portugal (e-mail: catalao@ubi.pt, sm@ubi.pt)

(2) Inst. Superior de Ingeniaría de Lisboa, Dpto. de Ingeniaría Electrotécnica y Automatización,

Calle Conselheiro Emídio Navarro, 1949-014 Lisboa-Portugal (e-mail: vfmendes@isel.pt)

(3) Inst. Superior Técnico, Dpto. de Ingeniaría Electrotécnica y Computadores,

Av. Rovisco Pais, 1049-001 Lisboa-Portugal (e-mail: Imf@ist.utl.pt)

\begin{abstract}
Resumen
El presente trabajo estudia el problema de la planificación de la operación de corto plazo de sistemas de energía termoeléctrica con restricciones ambientales. Las crecientes preocupaciones ambientales para limitar las emisiones de gases con efecto de estufa llevaron a la elaboración del Protocolo de Kyoto, introduciendo nuevas restricciones en la operación de los sistemas de energía termoeléctrica. Las herramientas tradicionales de apoyo a la decisión deben ser adaptadas a esta nueva coyuntura. El problema consiste en la determinación del plan de acoplamiento de las unidades y del perfil de producción mas adecuado para cada unidad térmica acoplada a lo largo del horizonte temporal. Para su solución, se considera un método de optimización basado en programación dinámica y programación no lineal, y se desarrolla un nuevo sistema de apoyo a la decisión en lenguaje Fortran que considera simultáneamente los costos de los combustibles y las emisiones. Se presentan y se discuten resultados de la simulación computacional a un caso práctico.
\end{abstract}

Palabras claves: optimización, simulación, energía termoeléctrica, restricciones ambientales

\section{Short-Term Operational Planning of Thermoelectric Power Systems with Environmental Constraints}

\begin{abstract}
This paper analyzes the problem of short-term operational planning of thermoelectric power systems with environmental constraints. The growing worldwide environmental concern to limit emission of greenhouse gases led to the Kyoto Protocol, introducing new constraints in the operation of thermoelectric power systems. The traditional tools that aid decision making must be adapted to this new framework. The problem consist of deciding the commitment of the units and the optimal generation profile for each thermal unit committed throughout the time horizon. For its solution, an optimization method based on dynamic programming and non-linear programming is considered, building a new decision support system in Fortran language which simultaneously considers fuel costs and emissions. The results of the computational simulation applied to a practical case are show and discussed.
\end{abstract}

Keywords: optimization, simulation, thermoelectric energy, environmental constraints 


\section{INTRODUCCIÓN}

En la gran mayoría de los países, entre los cuales Portugal, los combustibles fósiles son utilizados en porcentaje significativo para satisfacer sus necesidades energéticas. La producción de energía eléctrica por medio de centrales térmicas convencionales tiene efectos reconocidos como siendo nocivos para el medio ambiente (Bellhouse y Whittington, 1996). Estos efectos han originado preocupaciones en los niveles político y empresarial.

A nivel político estas preocupaciones han conducido a la elaboración del Protocolo de Kyoto, que para los países industrializados establece límites en la emisión de gases con efecto de estufa. Así mismo, a nivel empresarial las cuestiones ambientales constituyen hoy en día una ventaja para las empresas que aseguren la preservación del medio ambiente.

El Protocolo de Kyoto, siendo el principal acuerdo internacional para el combate a las alteraciones climáticas, entró en efecto después de la ratificación por la Federación Rusa a 16 de Febrero de 2005, siete años después de haber sido elaborado, terminando así la incertidumbre ocasionada en 2001 por la recusa de los Estados Unidos de América en ratificar el Protocolo, siendo los Estados Unidos el mayor emisor mundial de gases con efecto de estufa. Sin los Estados Unidos, el sí de la Federación Rusa fue vital para que se corroborasen los requisitos para la entrada en vigor del Protocolo: ser ratificado por 55 por ciento de los países signatarios, en los cuales estuvieran representadas 55 por ciento de las emisiones a nivel mundial en 1990, año base de referencia para el Protocolo de Kyoto. Con el sí de la Federación Rusa, el número de ratificaciones subió para 128 y las emisiones para 62 por ciento.

Portugal ya ha alcanzado en 2002 cerca del 41 por ciento de aumento en sus emisiones comparativamente con el año de 1990, siendo el límite establecido de 27 por ciento para el período 2008-2012. En consecuencia, Portugal tiene no solo que mejorar la eficiencia en la explotación de sus recursos hídricos (Catalão et al., 2006), como también tiene que considerar restricciones ambientales conjuntamente con la decisión de producción en sus centrales térmicas.
El problema de la planificación de la operación de sistemas de energía termoeléctrica se basa en dos procesos principales. El primero consiste en determinar los estados de acoplamiento de las unidades del sistema en cada periodo del horizonte temporal. Éste es el problema de la programación horaria. El otro proceso, llamado despacho económico, distribuirá la demanda de energía eléctrica entre las diferentes unidades acopladas. Esta distribución se llevará a cabo teniendo en cuenta las características de las centrales. Estos dos procesos están relacionados entre sí y sus salidas deben ser consideradas simultáneamente para obtener la solución óptima del problema.

Las nuevas restricciones ambientales han sido consideradas principalmente en el proceso de despacho económico (Abido, 2003; Dhillon y Kothari, 2000; Hobbs, 1993; Huang y Huang, 2003; Muslu, 2004), que pretende determinar únicamente el nivel de potencia de cada unidad, siendo el universo de decisión reducido a las unidades acopladas. El proceso de la programación horaria de centrales térmicas se ha resuelto típicamente para proporcionar el plan de acoplamiento de las centrales de forma que se suministre la demanda a un costo mínimo, no incorporando restricciones ambientales. En el pasado reciente la incorporación de las restricciones ambientales en este proceso no ha sido tan estudiada recientemente como en el proceso de despacho económico (Gjengedal, 1996; Kuloor et al., 1992). Sin embargo, la aparición del mercado de emisiones en la Unión Europea ha renovado el interés en este proceso (Lu y Shahidehpour, 2005; Pulgar-Painemal, 2005). $\mathrm{Si}$ no se consideran las restricciones ambientales en el problema de programación horaria, una unidad de costo reducido pero que emita más gases con efecto de estufa puede ser acoplada para despacho económico (Mendes et al., 2004).

En este artículo se propone un método de optimización usando programación dinámica y programación no lineal, que considera las emisiones en el problema de planificación de la operación de unidades térmicas. Se presenta un caso práctico con once unidades térmicas y un horizonte temporal de 168 horas, siendo comparados e discutidos los resultados obtenidos, sin y con la consideración de emisiones. 
La estructura de este artículo es la siguiente. En el apartado de la formulación se lleva a cabo la formulación matemática del problema. En el apartado del método de optimización se explica la aplicación de la programación dinámica y programación no lineal a este problema. En el apartado de resultados y discusión se presentan los resultados obtenidos en un caso práctico, y finalmente se recogen las conclusiones que se pueden extraer de este artículo.

\section{FORMULACIÓN}

El problema tradicional de la planificación de la operación de sistemas de energía termoeléctrica permite determinar el acoplamiento de unidades para la satisfacción de la demanda de energía eléctrica minimizando el costo total de operación, siendo las decisiones empleadas en intervalos de una hora a lo largo del horizonte temporal de un día hasta una semana.

El problema se formula como:

$\operatorname{Min} f(x, u, p)$

s.a:

$(x, u, p) \in F$

siendo la función objetivo dada por el costo total para la operación:

$f(x, u, p)=\sum_{k \in K} \sum_{i \in l} C_{i k}\left(x_{i k}, u_{i k}, p_{i k}\right)$

Con las nuevas restricciones ambientales, la función objetivo es la emisión total para la operación:

$f(x, u, p)=\sum_{k \in K} \sum_{i \in l} E_{i k}\left(x_{i k}, u_{i k}, p_{i k}\right)$

El costo total de operación es dado por el costo de arranque, considerado constante, y por el costo de combustible, aproximado por una función cuadrática:

$C_{i k}^{c o}\left(u_{i k}, p_{i k}\right)=u_{i k}\left(\alpha_{i}+\beta_{i} p_{i k}+\gamma_{i} p_{i k}^{2}\right)$

donde $\alpha_{i}, \beta_{i}$ y $\gamma_{i}$ son los coeficientes que determinan el costo de combustible para la unidad $i$.
El nivel de emisión es aproximado del mismo modo por una función cuadrática:

$E_{i k}^{e m}\left(u_{i k}, p_{i k}\right)=u_{i k}\left(a_{i}+b_{i} p_{i k}+c_{i} p_{i k}^{2}\right)$

donde $a_{i}, \quad b_{i}$ y $c_{i}$ son los coeficientes que determina la emisión para la unidad $i$.

El problema es sujeto a restricciones de carga y restricciones técnicas, que definen el conjunto de decisiones admisibles.

Las restricciones de carga pueden ser clasificadas en:

i) Restricciones de demanda, es decir, la suma de la potencia eléctrica de las unidades debe igualar la demanda de energía eléctrica en cada periodo:

$\sum_{i \in l} p_{i k}=D_{k}$

ii) Restricciones de reserva rodante, para mantener un cierto nivel de seguridad:

$\sum_{i \in A_{j k}} F_{j i}\left(x_{i k}, p_{i k}\right) \geq F_{j k}^{r e q}$

iii) Restricciones de combustible ó emisiones a lo largo del horizonte temporal:

$\sum_{i \in B_{n}} \sum_{k \in K} H_{n i}\left(x_{i k}, u_{i k}, p_{i k}\right) \geq H_{n}^{r e q}$

Las restricciones técnicas propias de cada unidad son:

a) La función de transición de estado:

$$
x_{i, k+1}=G_{i k}\left(x_{i k}, u_{i k}\right)
$$

b) Las limitaciones en el nivel de potencia:

$\underline{p}_{i} \leq p_{i k} \leq \bar{p}_{i}$

c) La imposición de estados iniciales y finales:

$x_{i o} \in X_{i 0} \quad x_{i f} \in X_{i f}$

Las restricciones dadas por las expresiones (7) a (12) definen el conjunto de operaciones posibles $F$. 
Desde un punto de vista matemático, este problema es combinatorio, no lineal y enteromixto, con variables enteras asociadas a estados discretos de las unidades y con variables continuas asociadas a los niveles de potencia de las unidades.

En cada periodo y para cada unidad existen dos estados posibles: ó la unidad está en funcionamiento, ó la unidad esta parada. El concepto de estado está asociado al número de horas en funcionamiento ó de parada para cada unidad. Por ejemplo, sea: up el tiempo mínimo de funcionamiento; down el tiempo mínimo de parada; cool el tiempo que corresponde al enfriamiento total y asimismo al mayor costo de arranque que la unidad presenta.

El diagrama de transición de estado se presenta en la Fig. 1, siendo las horas de funcionamiento descritas por números enteros positivos e las horas de parada descritas por números enteros negativos.

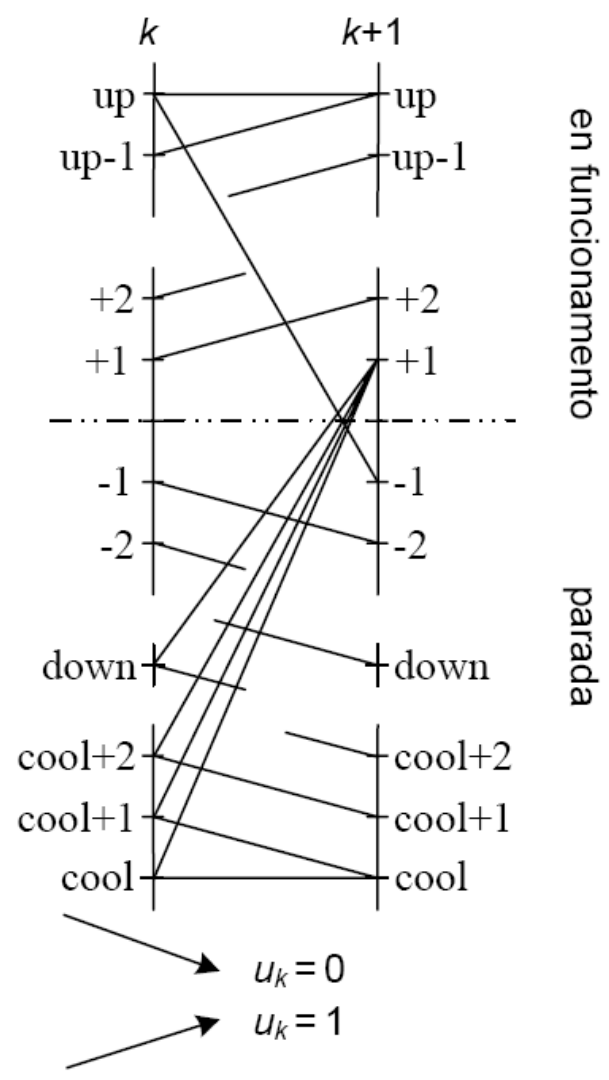

Fig. 1: Ejemplo de un diagrama de transición de de estado para una unidad

El número de estados de funcionamiento es igual a up e el número de estados de parada es igual a cool.
Así se tiene que:

Si el estado de la unidad es up, ella puede quedar en funcionamiento, ó ser desacoplada.

Si el estado de la unidad se encuentra entre el estado 1 e el estado up-1, la unidad debe quedar en funcionamiento.

Si el estado de la unidad se encuentra entre down+1 e -1, la unidad debe quedar parada.

Si el estado de la unidad es cool, la unidad puede permanecer parada ó ser puesta en funcionamiento.

\section{MÉTODO DE OPTIMIZACÍON}

La programación dinámica posibilita determinar cuales son las unidades que irán funcionar y cuando a lo largo del horizonte temporal.

La búsqueda de la solución óptima se organiza mediante un árbol de decisión, siendo el conocido teorema de Bellman el fundamento básico para este método. La programación dinámica se presta bastante bien para la optimización de procesos de múltiples etapas, como es el caso de la programación horaria.

Para las unidades en funcionamiento se tiene que determinar el nivel de potencia mas adecuado para la satisfacción de la demanda es decir, el despacho económico. Su resolución se hace empleando programación no lineal, cuja solución emplea un algoritmo de decisión del tipo:

Algoritmo calcular $p_{i k}^{*}$

Inicio

$p_{i k}^{*}=\left(\lambda_{k}-\beta_{i}\right) / \gamma_{i}$

Si $p_{i k}^{*}>\bar{p}_{i}$ entonces $p_{i k}^{*}=\bar{p}_{i}$

Si $p_{i k}^{*}<p_{i}$ entonces $p_{i k}^{*}=p_{i}$

Fin

siendo $\lambda_{k}$ el costo incremental en el periodo $k$ determinado por el despacho económico.

Información Tecnológica - Vol. 18 No 1 - 2007 


\section{RESULTADOS Y DISCUSIÓN}

La simulación computacional es basada en un caso práctico compuesto por once unidades térmicas, siendo el horizonte temporal de 168 horas.
En la Tabla 1 se muestran los coeficientes para las funciones cuadráticas que determinan el costo de combustible, dado por la expresión (5), y el nivel de emisión, dado por la expresión (6), para las once unidades térmicas consideradas.

Tabla 1: Coeficientes de costo y emisión.

\begin{tabular}{|c|c|c|c|c|c|c|c|c|}
\hline & \multicolumn{3}{|c}{ Costo } & $\underline{p}_{i}$ & $\bar{p}_{i}$ & \multicolumn{3}{c|}{ Emisión } \\
\hline$i$ & $\alpha$ & $\beta$ & $\gamma$ & $(\mathrm{MW})$ & $(\mathrm{MW})$ & $a$ & $b$ & $c$ \\
\hline 1 & 1675 & 18.78 & 0.013 & 60 & 300 & 25.8 & -0.52 & 0.007 \\
\hline 2 & 1207 & 18.96 & 0.018 & 60 & 300 & 26.9 & -0.54 & 0.007 \\
\hline 3 & 2277 & 19.71 & 0.010 & 50 & 500 & 30.1 & -0.49 & 0.004 \\
\hline 4 & 2239 & 21.02 & 0.009 & 50 & 460 & 30.1 & -0.39 & 0.004 \\
\hline 5 & 2292 & 20.84 & 0.010 & 50 & 500 & 25.3 & -0.56 & 0.004 \\
\hline 6 & 2516 & 19.78 & 0.012 & 50 & 500 & 25.3 & -0.53 & 0.004 \\
\hline 7 & 1895 & 20.86 & 0.019 & 20 & 215 & 23.9 & -0.40 & 0.008 \\
\hline 8 & 1860 & 22.00 & 0.015 & 20 & 210 & 23.9 & -0.40 & 0.008 \\
\hline 9 & 1410 & 20.39 & 0.049 & 20 & 250 & 31.6 & -0.63 & 0.004 \\
\hline 10 & 1270 & 17.92 & 0.077 & 20 & 250 & 34.3 & -0.68 & 0.004 \\
\hline 11 & 1469 & 19.71 & 0.077 & 20 & 210 & 22.9 & -0.64 & 0.005 \\
\hline & & & Total & 420 & 3695 & & & \\
\hline
\end{tabular}

La demanda de energía eléctrica a lo largo del horizonte temporal considerado se presenta en la Fig. 2.

En las Figs. 3 y 4 , respectivamente para las unidades térmicas 1 a 6 y para las unidades

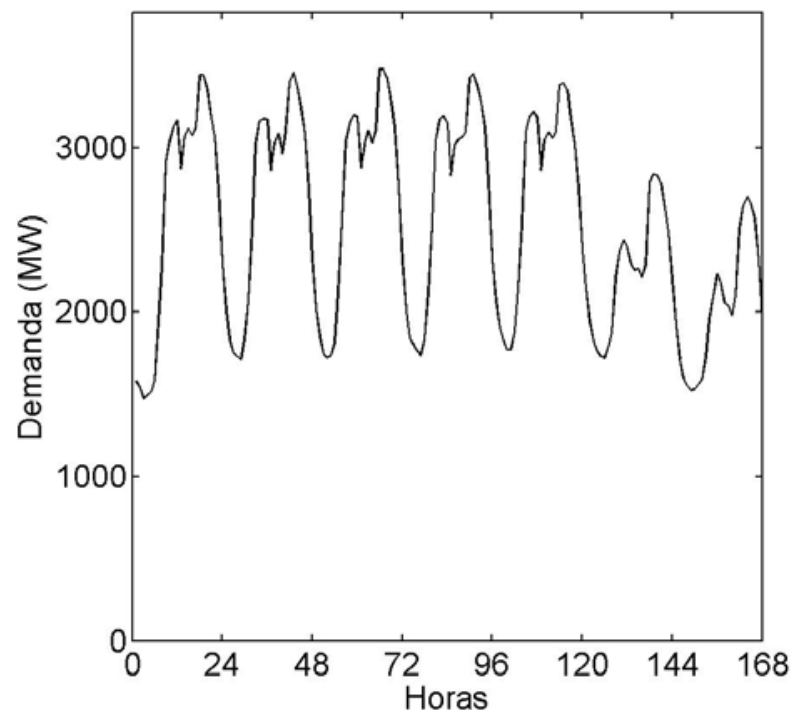

Fig. 2: Demanda horaria de energía eléctrica. térmicas 7 a 11, las líneas continuas representan la producción horaria sin la consideración de las emisiones y las líneas punteadas representan la producción horaria con la consideración de las emisiones.

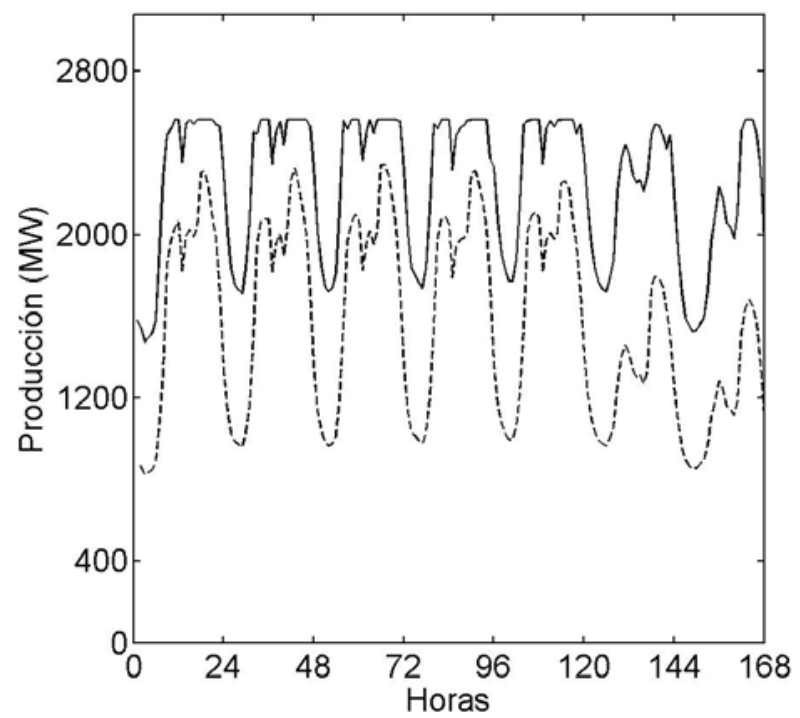

Fig. 3: Producción horaria de energía eléctrica para las unidades 1 a 6 . 


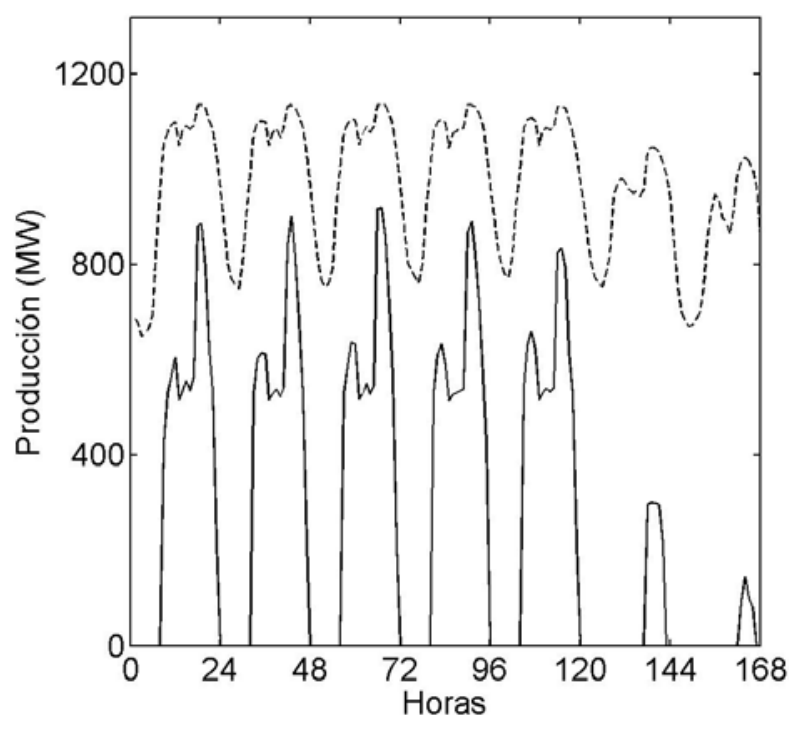

Fig. 4: Producción horaria de energía eléctrica para las unidades 7 a 11

Las unidades térmicas 1 a 6 tienen costos inferiores a los costos de las unidades térmicas 7 a 11, pero estas presentan emisiones más reducidas. Ignorando las emisiones, las unidades que presentan costos inferiores son acopladas típicamente a la potencia máxima, no siendo necesario acoplar todas las unidades para satisfacer la demanda.
El plan de acoplamiento de las unidades sin la consideración de las emisiones sigue el perfil de la demanda como se muestra en la Fig. 5. Contrariamente, con la consideración de las emisiones todas las unidades son acopladas, como se muestra en la Fig. 6, pero a un nivel inferior de potencia, lo que permite obtener un nivel de emisión mínimo. La emisión es un 42 por ciento inferior a la obtenida anteriormente, siendo el correspondiente aumento en el costo de solamente 12 por ciento.

En la Tabla 2 se muestran los resultados comparativos.

Tabla 2: Resultados comparativos.

\begin{tabular}{|c|c|c|c|}
\hline & $\begin{array}{c}\text { Costo } \\
\text { total (M) }\end{array}$ & $\begin{array}{c}\text { Producción } \\
\text { total (GW) }\end{array}$ & $\begin{array}{c}\text { Emisión } \\
\text { total }(\mathrm{Gg})\end{array}$ \\
\hline $\begin{array}{c}\text { Sin } \\
\text { emisiones }\end{array}$ & 12.99 & 425.61 & 600.92 \\
\hline $\begin{array}{c}\text { Con } \\
\text { emisiones }\end{array}$ & 14.61 & 425.61 & 348.33 \\
\hline
\end{tabular}

El tiempo de cálculo requerido para cada una de las planificaciones, sin y con emisiones, fue $2.7 \mathrm{~s}$, pudiendo ser considerado reducido.

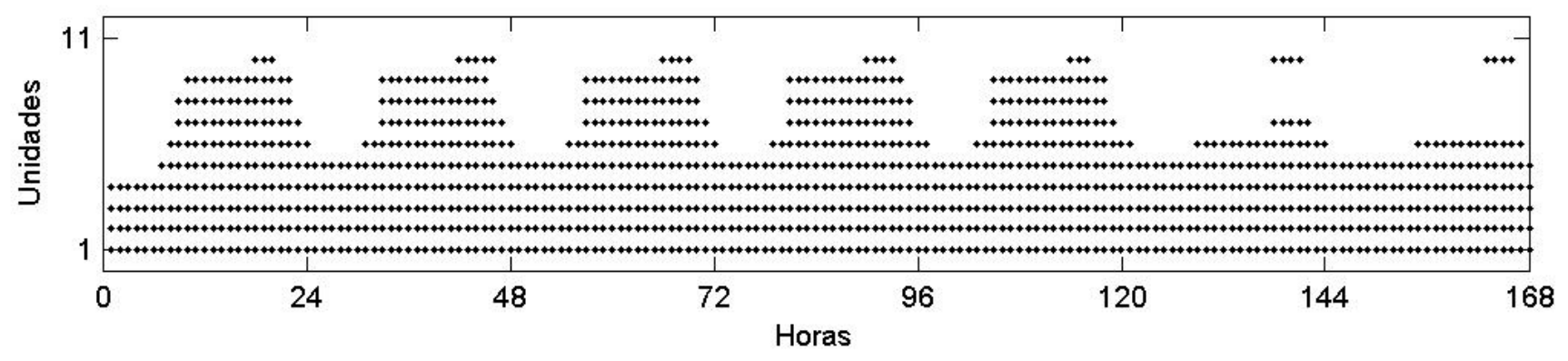

Fig. 5: Matriz de acoplamiento de las unidades a lo largo del horizonte temporal sin la consideración de las emisiones.

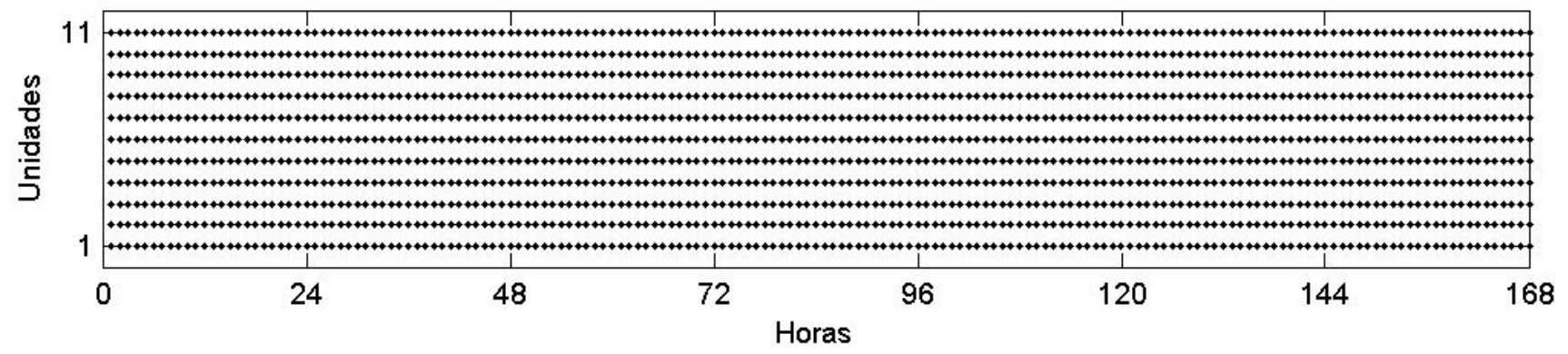

Fig. 6: Matriz de acoplamiento de las unidades a lo largo del horizonte temporal con la consideración de las emisiones. 


\section{CONCLUSIONES}

De los resultados presentados, de su análisis y de su discusión, se pueden obtener las siguientes conclusiones: 1) la característica principal de la formulación del problema de planificación de la operación de sistemas de energía termoeléctrica radica en considerar el costo de combustible en simultaneo con las emisiones; 2) una nueva herramienta de apoyo a la decisión ha sido implementada; y 3) el método propuesto es eficiente para obtener la producción horaria y el despacho económico con un tiempo de cálculo reducido.

\section{NOMENCLATURA}

I - Número total de unidades térmicas

J - Número total de restricciones por período

$N$ - Número total de restricciones a lo largo del horizonte temporal

$K$ - Número total de períodos

$D_{k}$ - Demanda de energía eléctrica en cada período $k$

$A_{j k}$ - Conjunto de unidades que tienen que satisfacer la restricción $j$ en cada período $k$

$B_{n}$ - Conjunto de unidades que tienen que satisfacer la restricción $n$ a lo largo del horizonte temporal

$C_{i k}$ - Costo total para la operación de la unidad $i$ en el período $k$

$E_{i k}$ - Emisión total para la operación de la unidad $i$ en el período $k$

$x_{i k}$ - Variable de estado para la unidad $i$ en el período $k$

$x$ - Vector de las variables de estado a lo largo del horizonte temporal

$X_{i 0}$ - Conjunto de los estados iniciales posibles para la unidad $i$

$X_{\text {if }}$ - Conjunto de los estados finales posibles para la unidad $i$

$u_{i k}$ - Variable entera de decisión para la unidad $i$ en el período $k$

$u$ - Vector de las variables enteras de decisión a lo largo del horizonte temporal

$p_{i k}$ - Variable de decisión para el nivel de potencia de la unidad $i$ en el período $k$

$p$ - Vector de la potencias de las unidades a lo largo del horizonte temporal

$F_{j i}$ - Contribución de la unidad $i$ para la restricción $j$

$H_{n i}$ - Contribución de la unidad $i$ para la restricción $n$
$G_{i k}$ - Función de transición de estado para la unidad $i$ en el período $k$

$\underline{p}_{i}, \bar{p}_{i}$ - Limites de potencia para la unidad $i$

\section{REFERENCIAS}

Abido, M.A., "A Niched Pareto Genetic Algorithm for Multiobjective Environmental/Economic Dispatch", Electrical Power and Energy Systems, 25(2), 97-105 (2003)

Bellhouse, G.M. y H.W. Whittington, "Simulation of Gaseous Emissions from Electricity Generating Plant", Electrical Power and Energy Systems, 18(8), 501-507 (1996).

Catalão, J.P., S.J. Mariano, V.M. Mendes y L.A. Ferreira, "Planificación de la Operación de Corto Plazo de Sistemas de Energía Hidroeléctrica", Información Tecnológica, 17(3), 149-155 (2006).

Dhillon, J.S. y D.P. Kothari, "The Surrogate Worth Trade-Off Approach for Multiobjective Thermal Power Dispatch Problem", Elec. Power Systems Research, 56(2), 103-110 (2000).

Gjengedal, T., "Emission Constrained UnitCommitment (ECUC)", IEEE Transactions on Energy Conversion, 11(1), 132-138 (1996).

Hobbs, B.F., "Emissions Dispatch Under the Underutilization Provision of the 1990 US Clean Air Act Amendments: Models and Analysis", IEEE Trans. Power Syst., 8(1), 177-183 (1993).

Huang, C. y Y. Huang, "A Novel Approach to Real-Time Economic Emission Power Dispatch", IEEE Trans. Power Syst. 18(1), 288-294 (2003).

Kuloor, S., G.S. Hope y O.P. Malik, "Environmentally Constrained Unit Commitment" IEE Proceedings-Generation Transmission and Distribution, 139(2), 122-128 (1992).

Lu, B. y M. Shahidehpour, "Unit Commitment with Flexible Generating Units", IEEE Trans. Power Syst., 20(2), 1022-1034 (2005).

Mendes, V.M.F., S.J.P.S. Mariano, J.P.S Catalão y L.A.F.M. Ferreira, "Emission Constraints on Short-Term Schedule of Thermal Units", $39^{\text {th }}$ Int. Universities Power Eng. Conf., 1068-1072, Bristol, UK, Sept. 6-8 (2004).

Muslu, M., "Economic Dispatch with Environmental Considerations: Tradeoff Curves and Emission Reduction Rates", Electric Power Systems Research, 71(2), 153-158 (2004).

Pulgar-Painemal, H.A., "Short-Term Generation Scheduling under a SO2 Emissions Allowances Market", Electric Power Systems Research, 74(2), 257-265 (2005). 
Agosto 2007

\begin{tabular}{|c|}
\hline $\begin{array}{c}\text { Simposio de la IUTAM sobre Hinchamiento y } \\
\text { Contracción de Materiales Porosos }\end{array}$ \\
\hline $\begin{array}{l}6 \text { al } 10 \text { de Agosto de } 2007 \\
\text { Petrópolis - RJ - Brasil }\end{array}$ \\
\hline $\begin{array}{l}\text { Informaciones: } \\
\text { National Laboratory of Scientific Computation } \\
\text { swelling@lncc.br } \quad \text { http://www.Incc.br/swelling/ }\end{array}$ \\
\hline
\end{tabular}

Congreso Internacional CARS \& FOF 2007. 23er Congreso Internacional ISPE en CAD/CAM, Robótica y Fabricas del futuro

$$
\begin{gathered}
16 \text { al } 18 \text { Agosto de } 2007 \\
\text { Bogotá - Colombia } \\
\text { Informaciones: }
\end{gathered}
$$$$
\text { cars.fof07@umng.edu.co }
$$

http://www.umng.edu.co/www/section-3791.jsp\#11842

$11^{\text {a }}$ Conferencia Internacional sobre la Formación de

Interfaces en Semiconductores (ICFSI - 11)

19 al 24 de Agosto de 2007

Manaus, Amazonas, Brazil

$$
\text { Informaciones: }
$$

ERON@ICFSI2007.COM http://www.icfsi2007.com http://www.physicstoday.org/cal/viewbyuser.jsp?eid=1117

\begin{tabular}{|c|} 
56th Sesión del ISI \\
INTERNATIONAL STATISTICAL INSTITUTE \\
22 al 29 de agosto de 2007 \\
Lisboa - Portugal \\
Informaciones: \\
ivette.gomes@fc.ul.pt \\
evelised@aip.pt itp://www.isi2007.com.pt/isi2007/index.php \\
\hline
\end{tabular}

II Congreso Iberoamericano y IV Congreso

Argentino de Química Analítica, ExpoARPIA 2007

27 al 30 de Agosto de 2007

Buenos Aires-Argentina

$$
\text { Informaciones: }
$$

info@lederdenegri.com.ar

http://www.sai.com.ar/aaqa/actividades.html

\section{$12^{\circ}$ Congreso Brasileño sobre Síntesis Orgánica $\left(12^{\text {th }}\right.$ BMOS)}

27 al 31 de Agosto de 2007

Itapema-Santa Catarina - Brasil

Universidad Federal de Santa Catarina- UFSC bmos-12@qmc.ufsc.br http://www.qmc.ufsc.br/ bmos12/index.html

\section{Septiembre 2007}

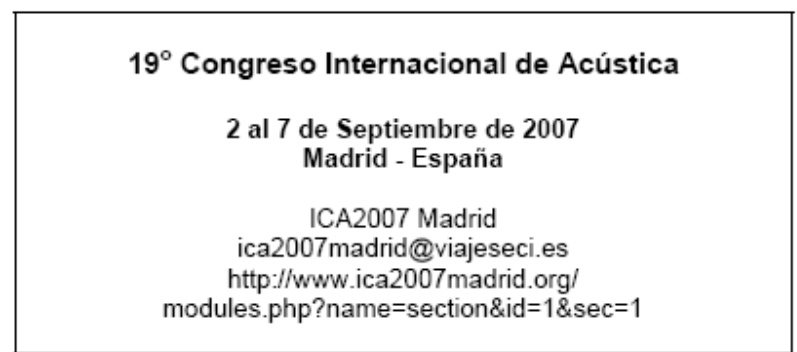

Simposio Internacional sobre Acústica Musical (ISMA2007)

9 al 12 de Septiembre de 2007 Barcelona - España

SEA, Serrano 144, 28006 Madrid, España isma2007barcelona@viajesci.es www.isma2007.org

9a Conferencia Internacional sobre Plasticidad Computacional, COMPLAS 2007

5 al 7 de Septiembre de 2007 Barcelona - España

Centro Int. de Mét. Numéricos en Ingeniería, Barcelona complas@cimne.upc.es

http://congress.cimne.upc.es/complas 07

XVII Congreso Latinoamericano de la Ciencia del Suelo, XVII CLACS

17 al 21 de Septiembre de 2007

León - Guanajuato - México

Sociedad Latinoamericana de la Ciencia del Suelo http://www.slcs.org.mx

e-mail: Ibrs@servidor.unam.mx; sicarubeu@hotmail.com

III Conferencia Internacional sobre Materiales

Textiles Compuestos y Estructuras Inflables

$$
\begin{aligned}
& 17 \text { al } 19 \text { de Septiembre de } 2007 \\
& \text { Barcelona - España }
\end{aligned}
$$

Centro Int. de Mét. Numéricos en Ingeniería, Barcelona membranes07@cimne.upc.edu

http://congress.cimne.upc.es/marine07

XIV Conferencia Europea sobre Mecánica de Suelos e Ingeniería Geotécnica

24 al 27 de Septiembre de 2007 Madrid-España

William Van Impe

ISSMGE y SEMSIG

http://www.ecsmge2007.org/ 